

AKADÉMIAI KIADÓ

UNIVERSITY of DEBRECEN

\section{International Review of Applied Sciences and Engineering}

12 (2021) 2, 119-126

DOI:

$10.1556 / 1848.2020 .00187$

(c) 2020 The Author(s)

\section{ORIGINAL RESEARCH} PAPER

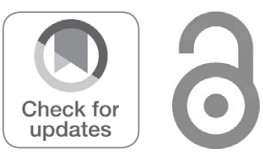

\title{
Inspection of transverse flaws for railways using phased array ultrasonic technique
}

\author{
Hajar Benzeroual $^{1 *} \odot$, Abdellatif Khamlichi ${ }^{2} \odot$ and Alia Zakriti ${ }^{2} \odot$ \\ ${ }^{1}$ Systems of Communication and Detection Laboratory, Faculty of Sciences, 93000 Tetouan, \\ Morocco \\ ${ }^{2}$ Department of Industrial and Civil Engineering Sciences, National School of Applied Sciences, \\ 93000 Tetouan, Morocco
}

Received: September 29, 2020 • Accepted: December 31, 2020

Published online: March 22, 2021

\begin{abstract}
Rail monitoring is an important activity which aims to preserve the safety and availability of railways. According to statistics, the primary cause of railway accidents is due to transverse defects that occur in the rail head. These special defects develop generally in a plane orthogonal to the rail running direction. The detection of these defects is a priority to increase the safety of rail transportation. Rail control monitoring techniques mostly rely on infrared thermography, eddy currents, air-coupled acoustic sensors, and ultrasounds. The present research studies the rail diagnosis by means of a non-contact device. The focus is on ultrasonic based methods where excitation is generated by thermal elastic coupling following laser irradiation of the rail head. For the reception of echoes, a special ultrasound sensor was used. In order to sense defects, phased array elements, which use multiple transducers and electronic time delays, are used to increase and to focalise the signal intensity. Flaws that have a moderate extension are better detected by the proposed method than with laser irradiation consisting of a single spot.
\end{abstract}

\section{KEYWORDS}

transverse flaws, phased array, ultrasound sensor, rotational laser vibrometer

\section{INTRODUCTION}

In railroad transportation, the progress in speed, axle loads and traffic frequency has had a direct consequence on increasing damage in the rails [1]. Damage ranges from internal cracks to surface defects and finally to complete fracture of the rail. Each type of defect is associated to a specific fatigue damage process; it has a specific lifetime and yields some impact on the safety of railways.

US Federal Railroad Administration's safety statistics [2] indicate that rail accidents arising from structures, roadbed, and track have been the source of nearly 4,300 derailments and near $\$ 845 \mathrm{M}$ in damage during the period between 2010 and 2017. The primary cause of those accidents was due to the "Transverse/compound fissure" and "Detail fracture - shelling/head check" appearing in rail head. These special defects are initiated from the rail head inside and propagate toward the head surface following generally an orthogonal plane to the rail running direction. They have been found to be responsible for 593 derailments and over $\$ 150 \mathrm{M}$ in cost, which represents nearly $20 \%$ of direct cost during the same period. For these reasons, rail inspection is an essential task, whose aim is to preserve the safety and availability of railway infrastructure.

Before the "Philadelphia Day Express" train derailment at Manchester, New York, on August 25, 1911, railways defect inspection had been performed visually [3]. Because of a transverse fissure, this accident has lead to the death of 29 persons and injuries of 60 others. Since then, many detection methods have been developed. 
Conventional inspection tests were carried out using excitation probes in contact with the rail head, such as pressure transducer, piezoelectric transducer, ultrasonic transducer or temperature transducer. The problem with this type of transducers is that it uses sliding probes, which are in contact with the rail head $[4,5]$. With this type of testing, the speed of inspection cannot exceed $50 \mathrm{~km} / \mathrm{h}$. Moreover, contact transducers cannot detect surface echoes due to the high noise level arising from the difficulty of reaching acoustic coupling among rail and sensor [4]. The technical limitation of contact excitation probes was the reason that has motivated researchers to consider that elimination of rail contact could permit detection of flaws under high speed. This has opened the possibility to explore enhanced ways of flaw detection techniques. Contactless rail inspection methods have relied mainly on eddy currents, Xrays, or infrared thermography [6-8].

The eddy currents method consists of the measurement of a material's response to electromagnetic fields over a specific frequency range [9]. In railroad track applications, eddy currents probes are located at a constant distance from the top railhead [10]. The main weakness of this method is its weak reliability in the particular case of a rail section, which is not completely broken, because the impedance may not allow getting a significant measurement.

The X-rays method is one of the few non-destructive evaluation methods that can examine the interior of an object. It belongs to the family of radiographic techniques that are the only methods that work on all materials. However, health and safety are the main X-rays method drawbacks. Also, the problematic of the portability of the Xrays equipment makes that it is used only for verification in places where defects have already been detected [11].

Comparing thermal properties between the defect and the host structure allows experienced users of infrared thermography method to notice the presence of anomalies from the simple observation of a single frame or a video sequence of the temperature distribution of the structure's surface. Infrared thermography provides a non-contact method that is able to accomplish full-field defect imaging of almost any material [12]. In the field of detecting rail defects, the infrared thermography approach has numerous limits such as unavailability of good area of viewing during motion and the presence of contaminants on the railroad surface, like dirt and grease, which causes varying cleanliness levels of the surface.

Years passed and rail traffic technology has undergone better development: rail traffic loads got heavier and speeds got higher. Hence the necessity for a more efficient and quicker method of flaws detection has been perceived. Modern inspection methods use contactless devices. The non-contact ultrasonic systems have proven to be optimal and suitable for several applications that contain the scan of wheels and railroads [13]. Among the field implemented ultrasonic non-contact systems for railways inspection one finds electromagnetic acoustic transducers (EMATs) based rail test vehicle [14], as well as prototypes relying on laser excitation and air-coupled acoustic sensors for acoustic echo reception [5, 15]. Systems with both laser excitation and laser reception were also proposed for general application use $[16,17]$. Recently, a device inspection configuration consisting of laser excitation and sensing through a 3D camera was proposed [18]. Using laser-based technology needs, however, some care for, at high-power level of the laser irradiation, the railroad surface may be ablated and plasma formed, causing melting and plastic deformation of the surface and even crack formation [19]. Laser irradiation should then be used at low energy density to avoid ablation and to obtain a thermo-elastic regime of deformation when directing the laser beam onto the railhead surface. The nonablative laser enables to generate ultrasonic waves consisting of bulk and surface waves which travel in the volume of the rail structure or at its surface.

In the previous works based on laser technology, laser excitation consisted of a single spot or of a static laser irradiation modulated through a slit mask. Because of the large effect of ambient perturbations and system uncertainties, the ratio of false predictions either positive or negative is expected to be high and more intense ultrasound waves are required to counteract that. To get intense waves in the non-ablative regime, phased arrays approach was considered. In the field of railways inspection, this idea was earlier presented in [20], where contact transducers coupled to the rail were used. Recently, a phased array laser ultrasonic method was applied to perform detection of defects in an elastic part while using a contact volumetric P-wave probe for reception [21].

The behaviour of laser-generated ultrasonic waves depends not only on the rail characteristics such as thermal conductivity, density, elasticity, and geometry of rail or depth but also on the parameters of the laser such as the spot size and pulse width of the laser beam [22]. This was the topic in a previous work [23], where the excitation was based on a focused laser beam on the surface of the railhead having the spot size $s=0.015 \mathrm{~m}$.

In this work, the focus is on ultrasonic based methods where excitation is generated by thermal elastic coupling following laser irradiation of the railhead. A contactless ultrasound sensor consisting of a rotational laser vibrometer that performs the reception of reflected echoes from defects is considered. By superposing individual spot responses, a phased array configuration can be achieved. The novelty of the proposed methodology for rail inspection consists of reaching intense wave excitation of the rail structure that involves only linear elastic ultrasounds that are generated in the non-ablative laser regime. It offers the possibility to steer and focalise the waves at a given depth location from the rail head surface.

\section{MATERIALS AND METHODS}

\subsection{Rail transverse defect inspection technique}

Transverse cracks in railways designate flaws having surface that develops vertically to the rail direction. The most 
common defects of this kind take the form of a transverse fissure, compound fissure or detail fracture. Transverse cracks are susceptible to lead to the weakening of tracks and they may also cause derailments $[24,25]$. The head of the rail is the part where transverse cracks show up most frequently and they can range from small invisible internal flaws to visible hairline surface defects [26]. The early detection of this kind of defect is then a priority to increase the safety of railways.

The ultrasonic wave generated by laser irradiation has longitudinal wave components propagating in railhead. To identify the presence of defects, various indicators based on the intensity of these ultrasound waves propagating through the rail structure have been proposed in the literature $[27,28]$.

The pulse duration of the excitation laser should be sufficiently short to capture all the echoes of interest by the detector. The use of a continuous laser is not a proper choice, since in this situation most of the light energy is obviously not used.

Detection of flaws in rails is monitored here through special ultrasound devices that generate waves at a given work frequency and sense the reflected echo, see Figs 1 and 2. The excitation method is based on surface-generated laser ultrasound by exposing a tiny spot to a short and concentrated laser pulse focused on the railhead. It is well known that the obtained volumetric wave propagation pattern is independent of the incidence angle of the exciting laser beam, which gives latitude of its orientation.

Therefore, at some location, which is difficult to access, the ultrasonic waves are emitted in almost all directions. The excitation transducer can consist of a single laser spot irradiation that excites the railhead. Since the energy of the laser spot should be intense enough and not exceeding the ablative threshold, to avoid the incidence of nonlinear waves which are difficult to interpret and which could damage the rail structure, a phased array (PA) strategy that involves multiple spotting at levels below the ablative limit is considered in the following. The lasers are fired sequentially according to time delays that will be fixed in order to maximize the wave intensity at some depth of the rail from the surface.

By the beginning of the 1990s, phased array technology was integrated as a new non-destructive evaluation method in ultrasonic datasheets and training manuals $[29,30]$. In the last decade, the applications of rail inspection using ultrasonic phased arrays were the main subject of a considerable number of researches in the world [31-33].

The PA approach enables to create intensively focused beams by constructive and destructive interference phenomenon. Unlike traditional single element ultrasonic transducers, the phased arrays method, which comprises multiple transducers, provides more flexibility by achieving beam steering without shifting the transducer. This enables a huge amount of information to be received by the rotational laser vibrometer with the only remaining complexity of its interpretation. However, new researches have emerged and deal with finding ways to make the process faster, such is the case of the Fast-Automated Angle Scan Technique.

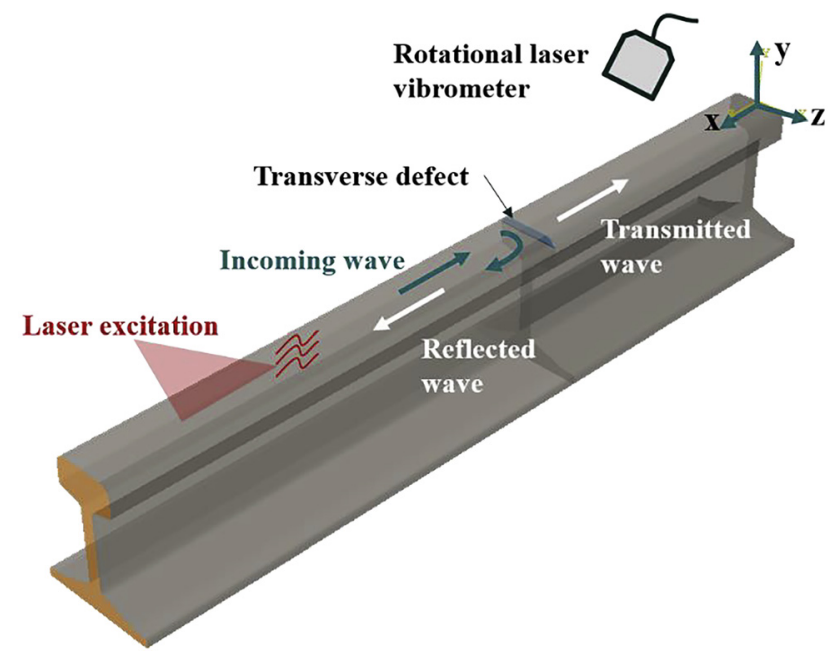

Fig. 1. Principle of detection of rail defect by using laser beam excitation and a rotational laser vibrometer sensor

Some solutions are already practicable. The French national railways (SNCF) uses an inspection method based on an ultrasonic phased array technique. The SNCF method uses multiple linear phased arrays contact transducer, counting 128 active elements [20]. The entire inspection can then be carried out by one single movement along the rail direction [34].

PA based technique is considered in this work, but use is made of non-contact laser elements according to the principle exposed in Figs 1 and 2.

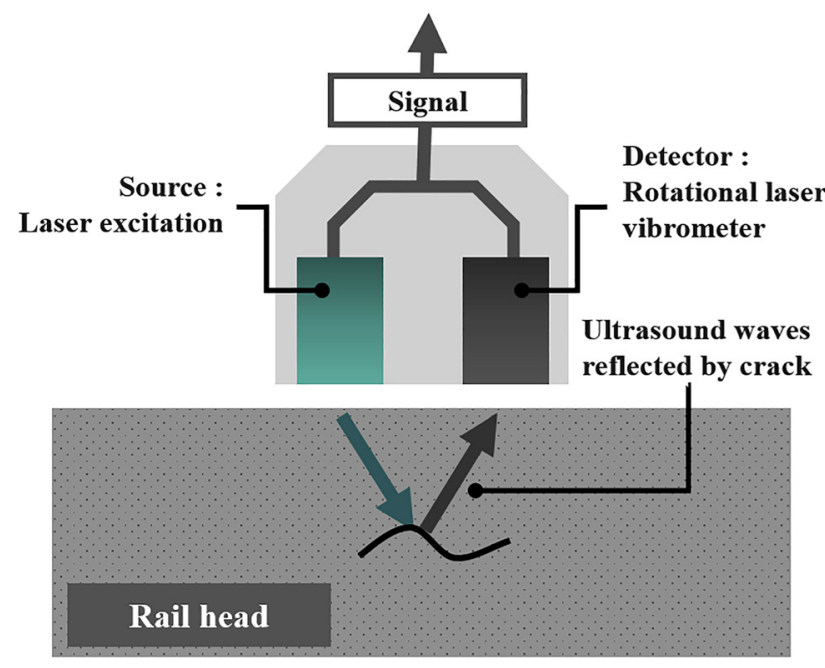

Fig. 2. Top view of the rail excitation and detection principle

Table 1. Material properties of $60 \mathrm{E} 1$ rail

\begin{tabular}{lc}
\hline Properties of 60E1 rail & Value \\
\hline Young's Modulus & $210 \mathrm{GPa}$ \\
Density & $7,800 \mathrm{~kg} / \mathrm{m} 3$ \\
Poisson's ratio & 0.3 \\
Thermal conductivity & $50.2 \mathrm{~W} / \mathrm{mK}$ \\
Specific Heat & $490 \mathrm{~J} / \mathrm{kg} \cdot \mathrm{K}$ \\
\hline
\end{tabular}




\subsection{Modelling and simulation of the rail system}

Considering a standard rail such as the 60E1, modelling of the transient ultrasound problem was performed via the finite element method. The rail structure was assumed to be made from a homogeneous and isotropic linear elastic steel material and having a localized transverse defect. The material properties, namely, density, Young's modulus, Poisson's ratio, thermal conductivity, and specific heat are given in Table 1.

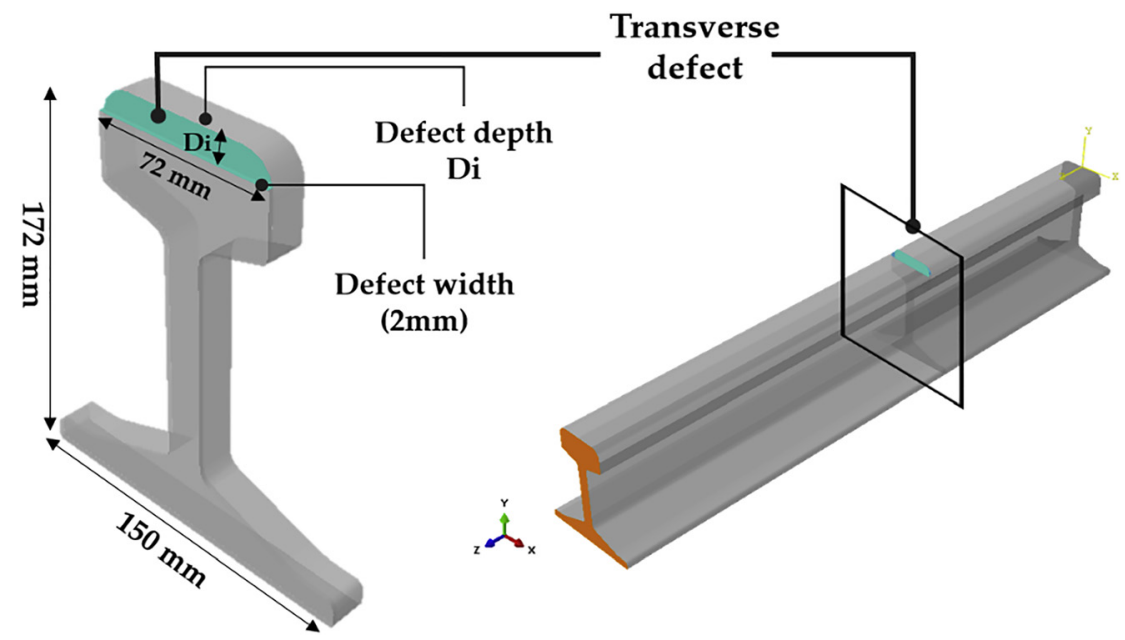

Fig. 3. Location and size of the considered transverse defect in a rail



Fig. 4. Position of the defect, sensors and the excitation spot area



Figure 5. The difference between the received signal at sensor 1 from a single spot excitation and that captioned after the phased array excitation is performed. 
The rail length considered in the finite element modelling is $400 \mathrm{~mm}$. The defect geometry is rectangular and has 2 $\mathrm{mm}$ in width with a variable depth (Di in $\mathrm{mm}$ ), see Fig. 3. In the simulations, adequate boundary conditions were used. Fig. 3 shows such a configuration.

The excitation is selected in such a way that it produces a wave field that is essentially non-ablative, and propagating in the volume and at the surface of the rail system. Such an excitation is provided by the special laser irradiation as discussed above.

Simulations were carried out for both intact rail and defected rail situations. They were performed by taking three levels of cut depth: 5, 10 and $20 \mathrm{~mm}$. The wave propagation phenomenon through the rail structure was first simulated under a single laser spot, then the other phases of excitation were added. The spot of the laser was applied on the

Table 2. The distances of considered spots zones and those of the sensors from the crack position according to the notations given in Fig. 4

\begin{tabular}{lc}
\hline Distances & Values \\
\hline $\mathrm{a}_{1}$ & $14 \mathrm{~mm}$ \\
$\mathrm{a}_{2}$ & $24 \mathrm{~mm}$ \\
$\mathrm{a}_{3}$ & $34 \mathrm{~mm}$ \\
$\mathrm{~b}_{1}$ & $14 \mathrm{~mm}$ \\
$\mathrm{~b}_{2}$ & $19 \mathrm{~mm}$ \\
$\mathrm{~b}_{3}$ & $24 \mathrm{~mm}$ \\
$\mathrm{~b}_{4}$ & $29 \mathrm{~mm}$ \\
\hline
\end{tabular}


Fig. 6. The first positive peaks of the observed signal at a given sensor location as function of the considered crack depth

railhead. The pulse width of the laser beam was selected to be $s=0.005 \mathrm{~m}$ and the pulse duration was set at $\Delta \mu=500$ ns. Each sensor uses the principle of laser vibrometry to determine the normal velocity component at some specific point in the rail. Four positions of implementing a rotational laser vibrometer sensor were analysed. Fig. 4 provides the arrangement of the chosen sensors in order to measure the deformation induced by the propagation of ultrasonic waves.

\section{RESULTS AND DISCUSSION}

The detection of rail defects was considered through special indicators, based on the variation of the intensity of the ultrasonic wave signal received during the rail monitoring operation. A FE approach of the suggested diagnostic device was developed using ABAQUS software. The model was simulated with three different depth defects located between the excitation spots area and the sensors.

The amplitude variability was investigated during the study as function of the sensor position and time of travel of the observed signal. In order to maximize the amplitude of the signals, a phased array technique was applied. This was achieved by using the principle of superposition. The actual PA excitation input of the device can be viewed to result from the summation of individual excitation spots.

The resulting signals after applying the phased array method showed that the signal is much more intense than that associated with a single spot excitation. As it can be seen 
in Fig. 5, the amplitude of a single spot (spot 1, spot 2 and spot 3) is inferior to that of the combined spots and this holds for all the different cases of defect depth. The considered location of the chosen spots is that which is detailed in Table 2 and Fig. 4.

Four points were selected on the surface of the railhead to observe the wave signal emitted by using phased array excitation technique. This was simulated by superposing individual responses of the laser spot excitation. The obtained responses of the system are plotted in Fig. 6. These results are obtained by performing the sum of measured responses due to the individual sensors that are associated to each single excitation spot. To enable detection and prevent the perturbation resulting from artificially reflected waves, an algorithm has been developed in MATLAB software to select only the first positive parts of the received signals, as shown in Fig. 6. The location of the selected sensors is indicated in Table 2 and Fig. 4.
The sensors used here sense the normal velocity to the rail surface at each considered sensor position. It is useful to identify the sensor's best position enabling to capture comprehensible information in order to determine the number adequate of sensors to be used in practice. From Fig. 6, one can see that as the depth of the transverse defect increases, the effect on the amplitude for a given sensor tends to decrease. As presented in Fig. 7, the obtained results indicate that the depth of the transverse defect affects the amplitude significantly. In order to separate between each crack depth, a satisfactory configuration of sensors is required. To determine that, an indicator based on the difference between flawed rail responses for consecutive crack depths was introduced. This enables to get a clear image of the observed defect signal evolution. Fig. 8 shows the obtained results in terms of achieved percentages.

This figure shows that the 5 and $10 \mathrm{~mm}$ crack depths can be easily identified by using sensors 1 and 2 , while

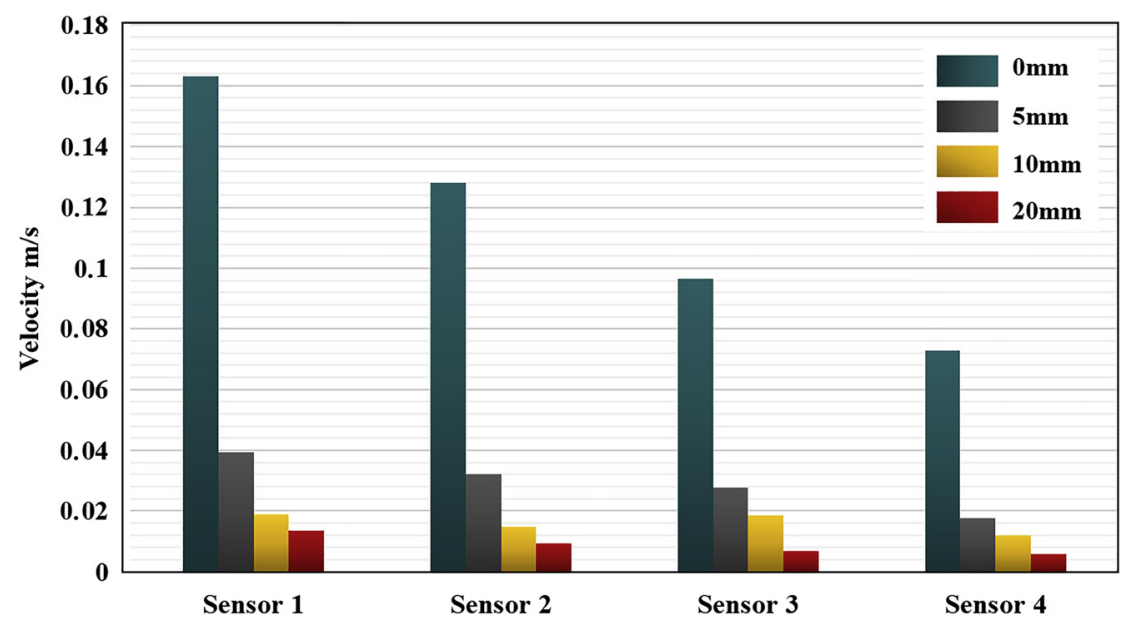

Fig. 7. The effect of the crack depth on the received amplitude by each sensor

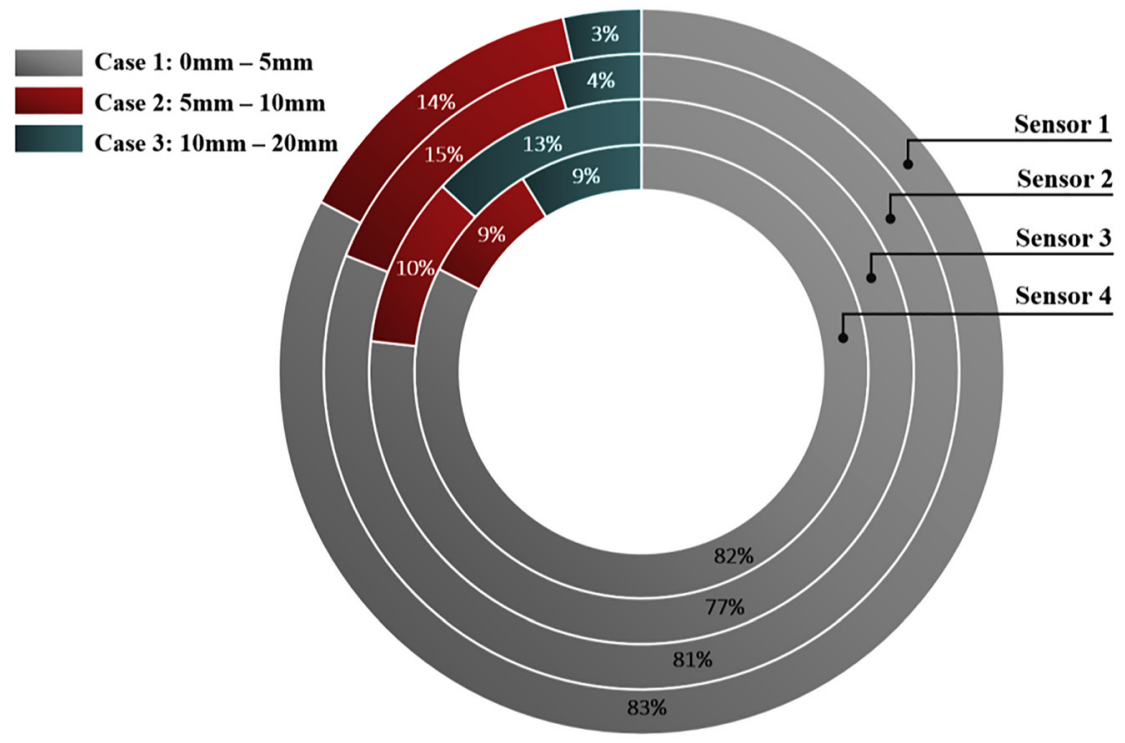

Fig. 8. The ability of each sensor to distinguish between defects of different depths as function of the considered sensor 
distinguishing between the $10 \mathrm{~mm}$ and $20 \mathrm{~mm}$ defect depths is more difficult for those sensors. In the case of sensor 4 , the indications associated to cases 2 and 3 are the same, which makes the difference between the crack depths of $10 \mathrm{~mm}$ and $20 \mathrm{~mm}$ not obvious to capture. On the other hand, the sensor 3 has the ability to clearly show the change for all situations, and the three depths of the defect can be well distinguished.

It should be noticed that from a practical point of view, if some dirt is present on the rail head like grease, clay or asphalt, with perfect contact between dirt and rail steel, then the proposed method will continue to be valid. Analysis of heat transfer problem in this situation by considering dirt thickness, thermal conductivity and thermal effusivity has shown that heat flux entering a rail head, made of steel, at the laser spot location is almost insensitive to the presence of dirt of this kind. Comparing the two situations with dirt and without dirt, the temperature variation was found to be of the order of $\Delta \mathrm{T}=0.1 \mathrm{dW}$, where $\mathrm{d}$ is the dirt thickness and $\mathrm{W}$ is the laser power. This is due to the high effusivity of steel, which is equal to $14000 \mathrm{~J} \cdot \mathrm{K}^{-1} \cdot \mathrm{m}^{-2} \cdot \mathrm{s}^{-1 / 2}$, while that of common dirt is of the order of $1200 \mathrm{~J} \cdot \mathrm{K}^{-1} \cdot \mathrm{m}^{-2} \cdot \mathrm{s}^{-1 / 2}$. However, if dirt is not in perfect contact with the rail steel material, such as, for example, is the case with a tree leaf with laminar air existing between dirt and the rail head, an important drop of temperature will occur; yielding that the method is no more applicable in this situation. So, cleaning the rail by sweeping its head is required in order not to detect such artificial and non pertinent defects. Furthermore, signal post-processing could be considered to filter the parasitic dirt signals, but this has still to be investigated.

\section{CONCLUSIONS}

In this work, the identification of transverse defects in the railhead was achieved by considering an integrated contactless system using laser excitation and motion sensing. A rail system simulation was carried out by using the finiteelement approach for the test configuration, where the system was exposed to ultrasounds generated through phased array elements technique.

Adequate position of a rotational laser vibrometer sensor has been determined. Detection of transverse rail flaws using a rotational laser vibrometer sensor has been analysed through monitoring indications using variations of the amplitude of the sensed speed. It was found that the proposed method allows the identification of defects that have a moderate extension better than with a single spot laser irradiation. The obtained results have made it possible to determine the best location of sensors to identify the transverse rail defects.

As future work, it would be interesting to develop a prototype based on the laser technology presented in this work, in order to achieve detection of flaws in real situations. The theoretical background anticipated in this work can be helpful for the design of such a system.

\section{REFERENCES}

[1] L. Oukhellou, E. Côme, L. Bouillaut, and P. Aknin, "Combined use of sensor data and structural knowledge processed by Bayesian network: Application to a railway diagnosis aid scheme," Transp. Res. Part C Emerg. Technol., vol. 16, no. 6, pp. 755-67, 2008. https://doi.org/10.1016/j.trc.2008.04.001.

[2] Federal Railroad Administration, Safety Statistics Data: 20102017, U.S. Department of Transportation, 2018. https://safetydata. fra.dot.gov/OfficeofSafety/default.aspx.

[3] Ogdensburg Advance and St. Lawrence Weekly Democrat: New York, 1911. https://nyshistoricnewspapers.org/lccn/sn83031423/ 1911-05-04/ed-1/seq-2/.

[4] H. Cygan, P. Aknin, P. Simard, and L. Girardi, "B-scan ultrasonic image analysis for internal rail defect detection," in World Congress on Railway Research, 2003. https://www.semanticscholar. org/paper/B-scan-ultrasonic-image-analysis-for-internal-railCygan-Girardi/c023bec9006ac80247284addee1a78cd04bab22b.

[5] F. Scalea, P. Rizzo, S. Coccia, I. Bartoli, M. Fateh, E. Viola, and G. Pascale, "Non-contact ultrasonic inspection of rails and signal processing for automatic defect detection and classification," Insight-NDT Cond. Monit. Spec. Issue NDT of Rails, vol. 6, no. 45, pp. 346-53, 2005. https://doi.org/10.1784/insi.47.6.346.66449.

[6] H. A. Toliyat, K. Abbaszadeh, M. M. Rahimian, and L. E. Olson, "Rail defect diagnosis using wavelet packet decomposition," IEEE Trans. on Industry Appl., vol. 39, no. 5, pp. 1454-61, 2003. https:// doi.org/10.1109/TIA.2003.816474.

[7] D. Cerniglia, G. Garcia, and S. Kalay, "Application of laser induced ultrasound for rail inspection," in World Congress on Railway Research, 2006.

[8] K. Labropoulos, P. Moundoulas, and A. Moropoulo, "Methodology for the monitoring, control and warning of defects for preventive maintenance of rails," in Tenth International Conference on Computer System Design and Operation in the Railway and Other Transit Systems, 2006, pp. 935-44. https://doi.org/10.2495/ CR060911.

[9] P. J. Shull, Nondestructive Evaluation: Theory, Techniques, and Applications, New York: Marcel Dekker, 2002. https://doi.org/10. 1201/9780203911068.

[10] H. M. Thomas, T. Heckel, and G. Hanspach, "Advantage of a combined ultrasonic and eddy current examination for railway inspection trains," Proc. ECNDT, vol. 49, pp. 341-4, 2006.

[11] G. A. Offereins and P. J. Mutton, "Recent experiences with the performance of aluminothermic rail welds under high axle loads," Proc. 13th Intl. Rail Track Conf., 2001.

[12] A. Vandone, P. Rizzo, and M. Vanali, "Two-stage algorithm for the analysis of infrared images," Res. Nondestructive Eval., vol. 23, no. 2, pp. 69-88, 2012. https://doi.org/10.1080/09349847.2011. 630557.

[13] R. E. Green, Jr, "Non-contact ultrasonic techniques," Ultrasonics, vol. 42, pp. 9-16, 2004. https://doi.org/10.1016/j.ultras.2004.01.101.

[14] S. Coccia, R. Phillips, I. Bartoli, S. Salamone, P. Rizzo, and F.L. di Scalea, "Online High-Speed Rail Defect Detection, Part II",DOT/ FRA/ORD-12/02, U.S. Department of Transportation, Federal Railroad Administration, Office of Railroad Policy and Development, Washington, DC, 2012, 20590 https://rosap.ntl.bts.gov/ view/dot/24200. 
[15] Y. Fan, S. Dixon, R. Edwards, and X. Jian, "Ultrasonic surface wave propagation and interaction with surface defects on rail track head," Independent Non-Destructive Test. Eval., vol. 40, no. 6, pp. 471-7, 2007. https://doi.org/10.1016/j.ndteint.2007.01.008.

[16] N. Montinaro, G. Epasto, D. Cerniglia, and E. Guglielmino, "Laser ultrasonics for defect evaluation on coated railway axles," NDT \& E Int., vol. 116, p. 102321, 2020. https://doi.org/10.1016/j.ndteint. 2020.102321.

[17] N. Montinaro, G. Epasto, D. Cerniglia, and E. Guglielmino, "Laser ultrasonics inspection for defect evaluation on train wheel," NDT \& E Int., vol. 107, p. 102145, 2019. https://doi.org/10.1016/j. ndteint.2019.102145.

[18] F. Masurkar, J. Rostami, and P. Tse, "Design of an innovative and self-adaptive-smart algorithm to investigate the structural integrity of a rail track using Rayleigh waves emitted and sensed by a fully non-contact laser transduction system," Appl. Acoust., vol. 166, p. 107354, 2020. https://doi.org/10.1016/j.apacoust.2020.107354.

[19] C. B. Scruby and L. E. Drain, Laser Ultrasonics: Techniques and Applications, Taylor \& Francis, 1990.

[20] P. Bredif, J. Plu, P. Pouligny, and C. Poidevin, "Phased-array method for the UT-inspection of French rail,". Repairs. Rev. QNDE, ed. by D. O. Thompson and D. E. Chimenti, vol. 27, pp. 762-9, 2008. https://doi.org/10.1063/1.2902739.

[21] F. Gao, H. Zhou, and C. Huang, "Defect detection using the phased-array laser ultrasonic crack diffraction enhancement method," Opt. Commun., vol. 474, p. 126070, 2020. https://doi. org/10.1016/j.optcom.2020.126070.

[22] P. Liu, N. Ab Wahab, and H. Sohn, "Numerical simulation of damage detection using laser-generated ultrasound," Ultrasonics, vol. 62, pp. 203-12, 2016. Available at: https://doi.org/10.1016/j. ultras.2016.03.013.

[23] H. Benzeroual, A. Khamlichi, and A. Zakriti, "Reliability of rail transverse flaw detection by means of an embedded ultrasonic based device," vol. 5, 2018. https://doi.org/10.1051/matecconf/201819100005.

[24] U. Zerbst, K. Madler, and H. Hintze, "Fracture mechanics in railway applications: An overview," Eng. Fracture Mech., vol. 72, pp. 163-94, 2005. https://doi.org/10.1016/j.engfracmech.2003.11.010.
[25] P. Rizzo, "Sensing Solutions for Assessing and Monitoring Railroad Tracks", Woodhead Publishing Limited, 2014. https://doi.org/ 10.1533/9781782422433.2.497.

[26] American Society for Nondestructive Testing, "Nondestructive testing handbook," Ultrason. Test., vol. 7, no. 2, pp. 284-97, 1991.

[27] A. Alemi, F. Corman, and G. Lodewijks, "Condition monitoring approaches for the detection of railway wheel defects," Proc. IMechE Part F: J. Rail and Rapid Transit., vol. 231, no. 8, pp. 961-81, 2017. https://doi.org/10.1177/0954409716656218.

[28] P. Rizzo, M. Cammarata, I. Bartoli, F. L. di Scalea, S. Salamone, S. Coccia, and R. Robert Phillips, "Ultrasonic Guided Waves-Based Monitoring of Rail Head: Laboratory and Field Tests", Hindawi Publishing Corporation, Advances in Civil Engineering, Article ID 291293, p. 13, 2010. https://doi.org/10.1155/2010/291293.

[29] J. Krautkramer and H. Krautkramer, "Ultrasonic testing of materials," Springer-Verlag, vol. 4, pp. 194-5, 1990. https://doi.org/10. 1007/978-3-662-10680-8.

[30] D. Utrata, "Exploring enhanced rail flaw detection using ultrasonic phased array inspection," Rev. QNDE, vol. 21, pp. 1813-8, 2002, Eds D. O. Thompson and D. E. Chimenti. https://doi.org/10.1063/ 1.1473013.

[31] D. Utrata, and R. Clark, "Groundwork for rail flaw detection using ultrasonic phased array inspection," Rev. QNDE, vol. 22, pp. 799-805, 2003, Eds D. O. Thompson and D.E. Chimenti. https://doi.org/10.1063/1.1570217.

[32] G. Garcia, and J. Zhang, "Application of ultrasonic phased arrays for rail flaw inspection," TTCI Rep. US Department Transportation, 2006.

[33] M. Ph. Papaelias, C. Roberts, and C. L. Davis, "A review on nondestructive evaluation of rails: State-of-the-art and future development," Proc. IMechE Part F: J. Rail and Rapid Transit., vol. 222, pp. 367-84, 2008. https://doi.org/10.1243/09544097JRRT209.

[34] P. Calmon, S. Mahaut, S. Chatillon, and R. Raillon, "CIVA: An expertise platform for simulation and processing NDT data," Ultrasonics, vol. 44, pp. 975-9, 2006. https://doi.org/10.1016/j. ultras.2006.05.218 